\title{
LINEARIZATION AND EXPLICIT SOLUTIONS OF THE MINIMAL SURFACE EQUATION
}

\author{
AleXANDER G. REZnIKOV
}

\begin{abstract}
We show that the apparatus of support functions, usually used in convex surfaces theory, leads to the linear equation $\Delta h+2 h=0$ describing locally germs of minimal surfaces. Here $\Delta$ is the LaplaceBeltrami operator on the standard two-dimensional sphere. It explains the existence of the sum operation of minimal surfaces, introduced recently. In 4-dimensional space the equation $\Delta h+2 h=0$ becomes inequality wherever the Gauss curvature of a minimal hypersurface is nonzero.
\end{abstract}

\section{Introduction}

Recently great progress was achieved in the investigation and construction of examples of minimal surfaces in $\mathbb{R}^{3}[\mathbf{1}]-[3]$. The Gauss map usually plays a significant role and its singularities in a sense control topology if the surface is complete [4]. It was also roticed [5], [6] that there exists a "sum" operation $M_{1}+M_{2}$ for two minimal surfaces $M_{1}$, $M_{2}$. It may seem to be strange, for the usual form of the minimal surface equation is essentially nonlinear. True, given a conformal minimal map $\mathbb{R}^{2} \supset U \stackrel{x}{\longrightarrow} M \subset \mathbb{P}^{3}$ we have a linear equation $\Delta x=0[7]$. However, the condition of conformality is nonlinear itself.

In this paper, we show that apparatus of support functions usually used in convex surface theory leads to the linear and completely integrable equation of minimal surfaces in $\mathbb{R}^{3}$. We are able to write down an explicit formula describing locally all minimal surfaces with nonvanishing curvature which is quite different from the Weierstrass description. We hope our method will be useful in global problems, too. It automatically implies the existence of the sum operation.

The main part of this work was done during the author's visit to Lithuania in 1987. I wish to thank Professor F. Weikša for fruitful discussions. I also wish to thank the referee for his very valuable rcmarks, in particular, for indicating to me that the relation of equation (6) to minimal surfaces was independently stated in [7]. 


\section{The main equation}

Let $M$ be a smooth oriented hypersurface in $\mathbb{R}^{N}$ and $G: M \rightarrow S^{N-1}$ be its Gauss map. Then [7] $G$ is a local diffeomorphism wherever the Gauss curvature of $M$ is nonzero. From now on, we assume that this condition holds at every point of $M$. Then $G$ becomes a covering over its image $G(M)$. Let $U \subset G(M)$ be a simply-connected proper domain, then $G^{-1}(U)$ is a disjoint union of open $V_{i}, i \in I$, and $\left.G\right|_{V_{i}}: V_{i} \rightarrow U$ is a diffeomorphism which we call $G_{i}$. We supply $S^{N-1}$ with the canonical metric of curvature 1.

Definition. By support function $h_{i}: U \rightarrow \mathbb{R}$ we mean

$$
h_{i}(n)=\left(G_{i}^{-1}(n), n\right) .
$$

Lemma 1. The function $h_{i}(n)$ determines $G_{i}^{-1}(n)$ in the following way:

$$
G_{i}^{-1}(n)=h_{i}(n) n+\operatorname{grad} h_{i}(n),
$$

where grad $h_{i}(n)$ is computed in terms of the metric of $S^{N-1}$ and looked at as a vector in $\mathbb{B}^{N}$.

Proof: Let $G_{i}^{-1}(n)=x$. For $y \in V_{i}$ we have by (1)

$$
h_{i}\left(G_{i}(y)\right)=\left(y, G_{i}(y)\right) .
$$

If $X \in T_{x} S^{N-1}$ then, differentiating (3) along $X$ we obtain

$$
\left(\operatorname{grad} h_{i}\left(G_{i}(x)\right), G_{i_{-}} X\right)=\left(X, G_{i}(x)\right)+\left(x, G_{i_{-}} X\right) .
$$

But $\left(X, G_{i}(x)\right)=0$ by the definition of the Gauss map, so

$$
\left(\left(x-\operatorname{grad} h_{i}\left(G_{i}(x)\right)\right), G_{i} X\right)=0 .
$$

By the nondegeneracy condition, $G_{i}$ maps isomorphically $T_{x} M$ onto $T_{G_{i}(x)} S^{N-1}$. The latter space coincides with $T_{x,} M$ as a subspace of $\mathbb{R}^{N}$ so, for some $\mu \in \mathbb{R}$, we have

$$
x-\operatorname{grad} h_{i}\left(G_{i}(x)\right)=\mu G_{i}(x),
$$

or

$$
G_{i}^{-1}(n)=\mu n+\operatorname{grad} h_{i}(n)
$$

Taking scalar product with $n$ and accounting $(1)$ and $\left(\operatorname{grad} h_{i}(n), n\right)=0$ we obtain (2). 
Lemma 2. Let $A(x)$ be the second fundamental operator in $T_{x} M$. Then

$$
A(x)=\left(h_{i}(n) E+\operatorname{Hess} h_{i}(n)\right)^{-1} .
$$

Here $n=G_{i}(x), E$ is the identity operator in $T_{n} S^{N-1}=T_{x} M$ and Hess $h_{i}(n)$ is the Hessian operator [8] on the sphere $S^{N-1}$.

Proof: Denote for a moment $F_{i}=G_{i}^{-1}$ on $U$. By (2) we have $F_{i}(n)=$ $h_{i}(n) n+\operatorname{grad} h_{i}(n)$. Let $Z \in T_{n} S^{N-1}$. By the definition of the Hessian operator, Hess $h_{i}(n)(Z)=\left(\nabla_{Z} \operatorname{grad} h_{i}\right)(n)$, where $\nabla$ is the Levi-Civita connection on the sphere. For any vector field $v$ on the sphere we have [8] $\nabla_{Z} v=v_{Z}^{\prime}+(v, Z) n$, where $v_{Z}^{\prime}$ denotes usual differentiation in $\mathbb{R}^{N}$. So by (2),

$$
\begin{aligned}
\left(\operatorname{Hess} h_{2}(n)\right)(Z) & =F_{i,} Z-\left(\operatorname{grad} h_{i}(n), Z\right) n-h_{i}(n) Z+\left(\operatorname{grad} h_{i}(n), Z\right) n \\
& =F_{i_{0}} Z-h_{i}(n) Z
\end{aligned}
$$

or $F_{i}, Z=h_{i}(n) Z+\left(\operatorname{Hess} h_{i}(n)\right)(Z)$. Let $Y=F_{z_{*}} Z$, then $Z=G_{*} Y$. By definition of $A, A(x) Y=G_{i_{*}} Y=Z$, which proves (5).

Theorem 1. Suppose $N=3$ and $M$ is minimal. Then for any proper simply-connected domain $U \subset G(M)$ and any branch $h_{i}$ we have

$$
\Delta h_{i}+2 h_{i}=0
$$

where $\Delta$ is the Laplace-Beltrami operator on the sphere $S^{2}$. Conversely, if $h$ is a solution of $(6)$ in an open $U \subset S^{2}$ then the formula $x(n)=$ $h(n) n+\operatorname{grad} h(n)$ determines a smooth map from $U$ to $\mathbb{B}^{3}$, which is either a constant or a conformal and minimal immersion outside a locally finite set of isolated singularities (branch points).

Proof: $M$ is minimal iff $\operatorname{tr} A(x)=0$ everywhere. For an invertible operator $A$ in 2-space we have $\operatorname{tr} A^{-1}=\frac{\operatorname{tg} A}{\operatorname{det} A}$ so by $(5), \operatorname{tr} A(x)=0$ is equivalent to $0=\operatorname{tr}\left(h_{2}(n) E+\right.$ Hess $\left.h_{i}(n)\right)=2 h_{i}+\Delta h_{i}$. This proves the first statcment of the theorem. Now suppose $h$ yields (6). Denote $F(n)=h(n) n+\operatorname{grad} h$. From the proof of the Lemma 2 we know that, $F_{*}(n)=h(n) E+$ Hess $h(n)$. In particular, it means that $F_{*}(n)$ maps $T_{n} S^{2}$ to itself and is symmetric in $T_{n} S^{2}$. Further, by $(6), \operatorname{tr} F_{*}(n)=$ 0 . Note that any symmetric operator with the zero trace in 2-space is represented by a matrix $\left(\begin{array}{cc}a & b \\ b & -a\end{array}\right)$ in any orthonormal basis and is thus conformal, so $F$ is conformal, and for $n \in U$ either rank $F_{*}(n)=2$ or $F_{*}(n)=0$. Denote by $Z$ the set of points where $F_{*}=0$. As $(6)$ is 
elliptic, $F(n)$ is analytic along with $h(n)$, so if $F$ is non-constant, then $Z$ is nowhere dense. Outside $Z, F(n)$ is a conformal immersion and we have just shown that $T_{F(n)} F(U)=T_{n} S^{2}$, so, for the Gauss map we have $G(F(n))=n$. Hence the support function $\tilde{h}$ of $F(U \sim Z)$ is $\tilde{h}(n)=(h(n) n+\operatorname{grad} h(n), n)=h(n)$. From the first part of the thcorem we see that $\left.F\right|_{U \sim Z}$ is minimal. It follows that $F$ is harmonic in $U \sim Z$, but $F$ is analytic in $U$ and $Z$ is nowhere dense, hence $F$ is harmonic everywhere in $U$. Locally in conformal coordinates $(x, y)$ we have $n \in Z \Leftrightarrow \frac{\partial F}{\partial x}=\frac{\partial F}{\partial y}=0$, hence $\mathcal{F}_{z}^{\prime}(n)=0$ where $z=x+i y$ and $\mathcal{F}$ is holomorphic and $\operatorname{Re} \mathcal{F}=F$, so $Z$ is locally finite.

Theorem 2. Suppose $N=4$ and $M$ is minimal. Then for any proper simply-connected $U \subset G(M)$ and any branch $h_{i}, \Delta h_{i}(n)+2 h_{i}(n)$ doesn't change sign in $U$.

Proof: Let $\lambda_{i}(x), 1 \leq i \leq 3$, be the principal curvatures of $M$ in $x$. Then $\lambda_{1}+\lambda_{2}+\lambda_{3}=0$ by minimality condition. Suppose $\Delta h_{i}(n)+$ $2 h_{i}(n)=0$. somewhere in $U$ and let $x=G_{i}^{-1}(n)$. As tr $A^{-1}(x)=$ $\frac{\lambda_{1} \lambda_{2}+\lambda_{1} \lambda_{3}+\lambda_{2} \lambda_{3}}{\operatorname{det} A(x)}$ we obtain by (5) that $\lambda_{1} \lambda_{2}+\lambda_{1} \lambda_{3}+\lambda_{2} \lambda_{3}=0$. This implies $\lambda_{1}^{2}+\lambda_{2}^{2}+\lambda_{3}^{2}=\left(\lambda_{1}+\lambda_{2}+\lambda_{3}\right)^{2}-2\left(\lambda_{1} \lambda_{2}+\lambda_{1} \lambda_{3}+\lambda_{2} \lambda_{3}\right)=0$ in $x$ which is impossible by nondegeneracy condition.

\section{Applications}

We turn to applications of our result. Let $M_{1}, M_{2}$ be two minimal surfaces in $\mathbb{R}^{3}$ such that $G\left(M_{1}\right) \cap G\left(M_{2}\right)$ has a nonempty interior in $S^{2}$. In [5] and [6] their sum $M_{1}+M_{2}$ is defined by parametrization $x(n)=G_{1}^{-1}(n)+G_{2}^{-1}(n)$. Let $h_{i}^{(1)}$ and $h_{j}^{(2)}$ be two branches of support functions of $M_{1}$ and $M_{2}$ respectively. Then by (2) we have $x(n)=$ $h(n) n+\operatorname{grad} h(n)$. where $h=h_{i}^{(1)}+h_{j}^{(2)}$. Next, both $h_{i}^{(1)}$ and $h_{j}^{(2)}$ yield (6) which is linear, hence $h$ yields (6), too. Theorem 1 implies thus the minimality of $M_{1}+M_{2}$. Moreover, given a minimal $M$ and any Killing vector field $Z$ in $S^{2}$ we can define the derivative surface $M_{Z}^{\prime}$ by

$$
h\left(M_{Z}^{\prime}\right)=h_{Z}^{\prime}
$$

which is also minimal. 
Example. Let $M_{1}$ be a catenoid defined in euclidean coordinates $(x, y, z)$ by the equation $x^{2}+y^{2}=(c h z)^{2}$. Then direct computations show that

$$
h_{1}(n)=h\left(n_{x}, n_{y}, n_{1}\right)=1-n_{Z} \arctan h n_{Z},
$$

and $G\left(M_{1}\right)=S^{2} \sim\{ \pm p\}$ where $p=(0,0,1)$. Let $g_{i} \in S O(3), i=$ $2, \ldots, m, g_{1}=i d$ be rotations such that the sets $\left\{ \pm g_{2} p\right\}$ are pairwise disjoint. Let $h=\sum h_{1} \circ g_{i}$, and $V=S^{2} \sim \bigcup_{i}\left\{ \pm g_{i} p\right\}$. Then we have the following

Proposition 1. The surface $\psi: n \mapsto h(n) n+\operatorname{grad} h$ is a complete minimal surfoce in $\mathbb{B}^{3}$ with only a finite number of branch points, and its Gauss image omits precisely $2 m$-point set $\bigcup_{i}\left\{ \pm g_{i} p\right\}$ (compare $\left.[5]\right)$.

Proof: First we note that in some neighbourhood of $p, \psi$ cannot have branch points. Indeed, suppose $\operatorname{det}(h(n) E+\operatorname{Hess} h(n))=0$, then for some vector $X \in T_{n} S^{2},\|X\|=1,\left(h_{1}(n) E+\right.$ Hess $\left.h_{1}(n)\right) X=$ $-\sum_{i \geq 2}\left(h_{i}(n) E+\right.$ Hess $\left.h_{i}(n)\right) X$. We know that for $i \geq 2 h_{i}(n)$ does not have singularities near $p$, so in some neighbourhood of $p$ and for some constant $C$ we would have

$$
\left\|\left(h_{1}(n) E+\operatorname{Hess} h_{1}(n)\right) X\right\| \leq C\|X\| .
$$

Denote $\left(h_{1}(n) E+\operatorname{Hess} h_{1}(n)\right) X=Y$, then $\|\left(h_{1}(n) E+\text { Hess } h_{1}(n)\right)^{-1} Y \| \geq$ $C^{-1}\|Y\|$. Actually $\left(h_{1}(n) E+\operatorname{Hess} h_{1}(n)\right)^{-1}$ is the second fundamental operator of the catenoid $M_{1}$, as we saw in Lemma 2, therefore, its eigenvalues are $\pm \sqrt{-K(n)}$, where $K(n)$ is the Gaussian curvature at $G^{-1}(n)$. As it is well-known (and easy to verify) that $K$ is decaying to zero at infinity, the above inequality is impossible. Of course, the same is true about all the singular points $\pm g_{i} p$, hence, being locally finite, the set of branch points should be finite. Next, as the metric of catenoid is complete, we have $\int_{\gamma}\left\|\left(h_{1}(n) E+\operatorname{Hess} h_{1}(n)\right) \dot{\gamma}(t)\right\|=\infty$ for any curve $\gamma:[0, \infty) \rightarrow S^{2}$ such that $\lim _{t \rightarrow \infty} \gamma(t)=p$. Hence the same arguments show that this is true for $h$ instead of $h_{1}$ and finally, $\psi$ is complcte.

Now consider the Enneper surface $[7] \varepsilon: \mathbb{R}^{2} \rightarrow \mathbb{P}^{3}$. The composition $G \circ \varepsilon$ with the Gauss map coincides with the inverse stercographic projection $\pi^{-1}: \mathbb{R}^{2} \rightarrow S^{2} \sim\{p\}$, so $K \neq 0$ and the support function $h$ is defined in $S^{2} \sim\{p\}$. Straightforward computations show that $K \underset{x \rightarrow \infty}{\longrightarrow}-0$ on $\varepsilon$, hence we can apply the same construction to obtain 
Proposition 2. For any given finite set $E \subset S^{2}$ there exists a complete minimal surface in $\mathbb{R}^{3}$ with only a finite number of branch points whose Gauss image omits precisely the set $E$.

The conjecture of Meeks $[4]$ states that for every $k>1$ there exists an embedded minimal surface homeornorphic to a compact manifold punctured in $k$ points. The problem of Osserman [7], solved by Fujimoto [4], asks whether the statement of our Proposition 2 holds for some smooth complete minimal surface (without branch points).

\section{Concluding Remarks.}

1. Our main equation (6) admits separation of variables. Fix $x_{0} \in S^{2}$, then in spherical polar coordinates near $(r, \varphi)$ near $x_{0}$ the spherical metric becomes $d r^{2}+\sin ^{2} r d \varphi^{2}$ and the LaplaceBeltrami operator becomes $\Delta f=f_{r}^{\prime}+\frac{\cos r}{\sin r} f_{r}^{\prime}+\frac{1}{\sin ^{2} r} f_{\varphi}^{\prime \prime}$. Substituting $f(r, \varphi)=\frac{1}{\sqrt{\sin r}} g(r, \varphi)$ we obtain that $\Delta f+2 f=0$ is equivalent to $g_{r}^{\prime \prime}+g\left(\frac{1}{4 \sin ^{2} r}+\frac{9}{4}\right)+\frac{g^{\prime \prime} \varphi}{\sin ^{2} r}=0$. By Fourier methods one finds

$$
g(r, \varphi)=\sum_{m=-\infty}^{\infty} C_{m}(r) e^{i m \varphi}
$$

where $C_{m}(r)$ satisfy

$$
C_{m}^{\prime \prime}+C_{m}\left(\left(\frac{1}{4}-m^{2}\right) \frac{1}{\sin ^{2} r}+\frac{9}{4}\right)=0 .
$$

2. A rather surprising phenomenon follows from our description. Namely, if $\Delta h+2 h=0$ in an open $U \subset S^{2}$, then the "MongeAmpere" $\psi=\operatorname{det}(h E+$ Hess $h)$ satisfies some second order $P \mathcal{D E}$. Indeed, we know from Thcorem I that the surface $M$ parametrized by $F(n): n \mapsto h(n) n+\operatorname{grad} h(n)$ is minimal and its curvature at $F(n)$ is $\psi^{-1}(n)$. Let's pull back on $S^{2}$ the minimal surface's metric. We will obtain $\hat{g}=\mu(n) g_{0}$, because the Gauss map $F^{-1}(n)$ is conformal (here $g_{0}$ is the spherical metric). To compute $\mu(n)$, we note that $F^{*} d s= \pm \psi(n) d s_{0}$, where $d s, d s_{0}$ are the area elements on $M, S^{2}$ respectively. Hence, $g=|\psi(n)| g_{0}$. Therefore, the curvature of $-\psi(n) g_{0}$ is $\psi^{-1}(n)$ (compare with Ricci-Curbastro Theorem, [10]). This is equivalent to some $P \mathcal{D} E$.

3. Suppose $M$ is a complete minimal surface of finite total curvature. Then by the theorem of Osserman [7] the Gauss map $G: M \rightarrow$ $S^{2}$ extends to a holomorphic map $\widetilde{G}$ of the completion $\widetilde{M}$, and 
$\widetilde{M} \sim M$ is finite, say $\widetilde{M} \sim M=\left\{p_{1}, \ldots p_{m}\right\}$. Let $\mathcal{L} \subset \vec{M}$ be the finite set of branch points of $\tilde{G}$, say $\mathcal{L}=\left\{q_{1} \ldots q_{k}\right\}$. Then $N=\widetilde{M} \sim((\widetilde{M} \sim M) \cup \mathcal{L})$ (is the finite covering of $S^{2} \sim \widetilde{G}((\widetilde{M} \sim$ $M) \cup \mathcal{L}$ ). Next, the support function $h(n)$ becomes single-valued on $N$ and we see that every complete minimal surface of finite total curvature determines a solution of the equation $\Delta h+2 h=0$ in a finite covering of the standard sphere punctured in a finite number of points.

4. Consider the flat metric $g=d x^{2}+d y^{2}-d z^{2}$ in $\mathbb{R}^{2,1}$. If for a surface $M \subset \mathbb{R}^{2,1},\left.g\right|_{M}$ is positively defined, then there exists a correctly defined Gauss map $G$ from $M$ to the hypcrboloid $S: x^{2}+y^{2}-$ $z^{2}=-1$. It is well-known that $g \mid S$ is the standard hyperbolic metric. Just as before we can define a support function $h(n)$. Formula (2) in this case reads $G_{i}^{-1}(n)=-h_{i}(n)+\operatorname{grad} h_{i}(n)$. Formula 5 becomes $A(x)=\left(-h_{i}(n) E+\operatorname{Hess} h_{i}(n)\right)^{-1}$ and $(6)$ becomes $\Delta h_{i}(n)-2 h_{i}(n)=0$ for minimal surfaces $M$ with timelike normals.

\section{References}

1. Costa C., Bull. Soc. Bras. Math. 15 (1984), 47-54.

2. Horrman D., Meeks W. III, Bull. Amer. Math. Soc. 12 (1985), 134-136.

3. Hoffman D., Meeks W. III, J. Differential Geom. 21 (1985), 109-127.

4. Fu」moro H., J. Math. Soc. Japan 40 (1988), 237-249.

5. Meeks W. III, "Proc. Int. Congr. Math. Berkeley 1986," 1987, pp. 551--558.

6. Rosenberg h. Toubiana E., J. Differential Geom. 28 (1988), $115-132$.

7. Langevin R., Rosenberg H., Duke Math. J. 57 (1988), 819-828.

8. Langevin R., Levitt G., RosenberG H., Banach center publ. 20 (1987).

9. Osserman R., "A Survey of Minimal Surfaces," Van Nostrand Reinhold Company, New York, Cincinnati, Toronto, London, Melbourne, 1969 . 
10. Gromoli D., Klingenberg W., Mryer W., "Riemannische Geometrie in "Großen," Lecture Notes in Math, 55, Springer, Berlin, Heidelberg, New York, 1968.

11. Lawson, H.B., JR., "Lectures on Minimal Submanifolds," Publish or Perish, 1980.

Permanent address:

School of Mathematical Sciences

Raymond and Beverly Sackier

Faculty of Exacl Sciences

Tel Aviv University

Rarnat-Aviv, Tel Aviv 69978

ISIRAEL
Current address:

International Centre for

Theoretical Physics

P.O.B. 586 Miramare

34100 Trieste

I'TALY

Primera versió rebuda el 29 d'Octubre de 1990 , darrera versió rebuda el 22 de Maín de 1991 